\title{
Health mindset is associated with anxiety and depression in patients undergoing treatment for breast cancer
}

\author{
Paulina M. Gutkin BS ${ }^{1}$ (D) | Katherine E. Fero MD $^{2}$ | Clare E. Jacobson BA ${ }^{3}$ | \\ Jie Jane Chen BA ${ }^{4}$ | Rachel V. Liang BA ${ }^{1}$ | Christopher Kolar MSEd ${ }^{5}$ | Rie von Eyben MS ${ }^{1}$ | \\ Kathleen C. Horst MD ${ }^{1}$ (D) \\ ${ }^{1}$ Department of Radiation Oncology, Stanford University School of Medicine, Stanford, California \\ ${ }^{2}$ University of California, Los Angeles, California \\ ${ }^{3}$ University of Michigan Medical School, Ann Arbor, Michigan \\ ${ }^{4}$ Harvard Medical School, Boston, Massachusetts \\ ${ }^{5}$ Independent Education Consultant, Palo Alto, California
}

Correspondence: Kathleen C. Horst, Department of Radiation Oncology, Stanford University School of Medicine, 875 Blake Wilbur Dr., Stanford 94305-5847, CA.

Email: kateh@stanford.edu

A person's mindset can affect their motivation, achievement, and well-being. ${ }^{1,2}$ However, the impact of mindsets in the health care setting is less established, particularly as it relates to a cancer diagnosis. We developed a novel measure to determine whether there is a distinct Health Mindset that impacts how patients address illness, focusing on those diagnosed with breast cancer. We tested the internal validity and reliability of the measure and assessed whether a patient's Health Mindset correlated with patient-reported measures of depression, anxiety, or insomnia.

Between March 2012 and October 2012, 50 consecutive women undergoing radiotherapy for breast cancer were enrolled in an Institutional Review Board-approved study. An additional 70 patients were enrolled between April 2017 and May 2019, for a total of 120 patients (ClinicalTrials.gov Identifier: NCT01553825). During the first and last weeks of radiotherapy, patients completed a survey consisting of four questionnaires:

1. Experimental questionnaire: Responses were recorded on a 6-point Likert-type scale, with questions reflecting whether cancer was seen as separate from their identity or whether their sense of self changed as a result of a cancer diagnosis. Those who scored 4 or higher, on average, were classified as having maintained their sense of self separate from the diagnosis; those who scored 3 or lower were classified as perceiving that their identity changed as a result of the cancer diagnosis.

2. The Hospital Anxiety and Depression Scale (HADS) ${ }^{3}$ : Responses were rated on a 4-point Likert scale with high scores indicating more anxiety or depression. A cutoff value of 8 or greater in each domain is indicative of clinically significant anxiety or depression.

3. The Insomnia Severity Index (ISI) ${ }^{4}$ : Responses were rated on a 5-point Likert scale, with high scores indicating more severe insomnia. A clinical cutoff score of 15 or more has been associated with clinical insomnia.

4. The Kind of Person Implicit Theory Scale (KOP) ${ }^{2}$ : Responses were recorded on a 6-point Likert-type scale. Scores of 4 or higher on average indicate a growth mindset with respect to their personality and traits; scores of 3 or less indicate a fixed mindset.

Ninety-seven patients (81\%) completed the survey at both time points for a total of 220 completed surveys. Two patients were enrolled but did not complete any surveys and were excluded from the final analysis.

The experimental questionnaire was analyzed using principal components analysis using data from cohort 1 . The analysis identified nine questions that accounted for $32 \%$ of the variability. These questions demonstrated strong factorial validity and defined our final Health Mindset Instrument (Figure 1). This novel instrument indicated strong internal consistency (Cronbach's alpha $=0.91$ ). A cross-validation leaving each individual item out and estimating the Cronbach's alpha on the remaining eight items resulted in alphas ranging from 0.89 to 0.91 , further confirming a strong internal consistency.

Anxiety, depression, and insomnia scores are depicted in Table 1. Notably, $31 \%$ of patients indicated clinically significant anxiety, and $11 \%$ of patients indicated clinically significant depression. Between 
1

2

3
4
5
6

\section{Strongly Agree Agree Mostly Agree Mostly Disagree Disagree Strongly Disagree}

\section{I often wonder whether I could ever get back to who I was before my cancer diagnosis.}

2. Despite my best efforts, from morning until night I think about how cancer has become who I am.

3. I may be minimizing things, but I really feel like my identity is intact.

4. I know I should not feel this way, but I feel like cancer has taken away my identity.

5. If I had to be completely honest, cancer dominates my view of myself.

6. Although I am a cancer patient, the cancer does not define who I am.

7. Despite my diagnosis, the cancer does not take away from who I am.

1 Extremely
2

Very Much
3

Moderately
4

Slightly
5

A little
6

Not at all

\section{To the extent that you feel cancer has changed who you are, how much has it been for the worse?}

\section{To what extent do you feel that your diagnosis has diminished who you are?}

FIGURE 1 Final Health Mindset Instrument

TABLE 1 Patient survey scores on the first and last days of radiation treatment

\begin{tabular}{|c|c|c|c|c|}
\hline & \multicolumn{2}{|l|}{ T1 score } & \multicolumn{2}{|l|}{ T2 score } \\
\hline & Mean (SD) & range & Mean (SD) & range \\
\hline Health mindset & $4.8( \pm 0.97)$ & $1.8-6$ & $4.9( \pm 0.99)$ & $1.8-6$ \\
\hline \multicolumn{5}{|c|}{ Hospital anxiety and depression } \\
\hline Anxiety & $6.5( \pm 3.4)$ & $1-16$ & $6.1( \pm 3.7)$ & $1-16$ \\
\hline Depression & $3.6( \pm 3.3)$ & $0-15$ & $3.4( \pm 3.1)$ & $0-15$ \\
\hline $\begin{array}{l}\text { Insomnia } \\
\text { severity index }\end{array}$ & $7.7( \pm 5.6)$ & $0-22$ & $8.0( \pm 6.1)$ & $0-24$ \\
\hline $\begin{array}{l}\text { Kind of person } \\
\text { implicit theory } \\
\text { scale }\end{array}$ & $3.3( \pm 0.8)$ & $1-6$ & $3.3( \pm 0.9)$ & $1.8-6$ \\
\hline
\end{tabular}

the first and last days of radiation treatment, the average mean score for ISI increased to 8, which is considered subthreshold insomnia. At the start of radiation therapy, $23 \%$ of patients identified as having a growth mindset, while $77 \%$ indicated a fixed mindset. This did not change over the course of radiation treatment.

Ninety-two patients (78\%) identified as having maintained their sense of self separate from the cancer diagnosis, while 26 patients (22\%) felt that the cancer diagnosis changed their identity. A multivariable model was fit to the data with Health Mindset as the outcome, with HADS, ISI, Kind of Person, and demographic characteristics (marital status, stage, chemotherapy use, and whether the patient was in the initial cohort) as predictors. Both anxiety $(P=.01)$ and depression $(P=.005)$ significantly correlated with Health Mindset. High scores on Health Mindset correlated with low scores on HADS, indicating that patients who had maintained a sense of self were less likely to have anxiety or depression (Figure 2). These results did not differ between cohort $1(n=50)$ and cohort $2(n=68)$. There was no significant correlation between Health Mindset and insomnia, marital status, stage of cancer, receipt of chemotherapy, or timing of survey. Furthermore, Health Mindset scores did not correlate with Kind of Person scores, suggesting that an individual's perception of themselves as having either a fixed or growth mindset does not influence the extent to which they identified with their breast cancer diagnosis. Lastly, the Health Mindset Instrument was evaluated to be independent of both the HADS-A and HADS-D instruments (Cronbach's alpha $=0.33$ ), confirming that the Health Mindset Instrument represents a unique entity.

Previous work focusing on cancer survivorship and a "cancer identity" demonstrates that patients may identify as a victim, patient, or survivor at various time points throughout the treatment course and follow-up period. ${ }^{5}$ This cancer identity can influence health-related quality of life and psychological well-being 5-15 years after the diagnosis. ${ }^{6}$

While our study is limited by small sample size and its exploratory nature, our study provides a unique instrument to measure the impact of a cancer diagnosis on a person's sense of self. Future studies will be important to replicate and confirm these results as well as introduce interventions that could modify a patient's mindset as a novel approach to addressing anxiety and depression during and after treatment for breast cancer. 


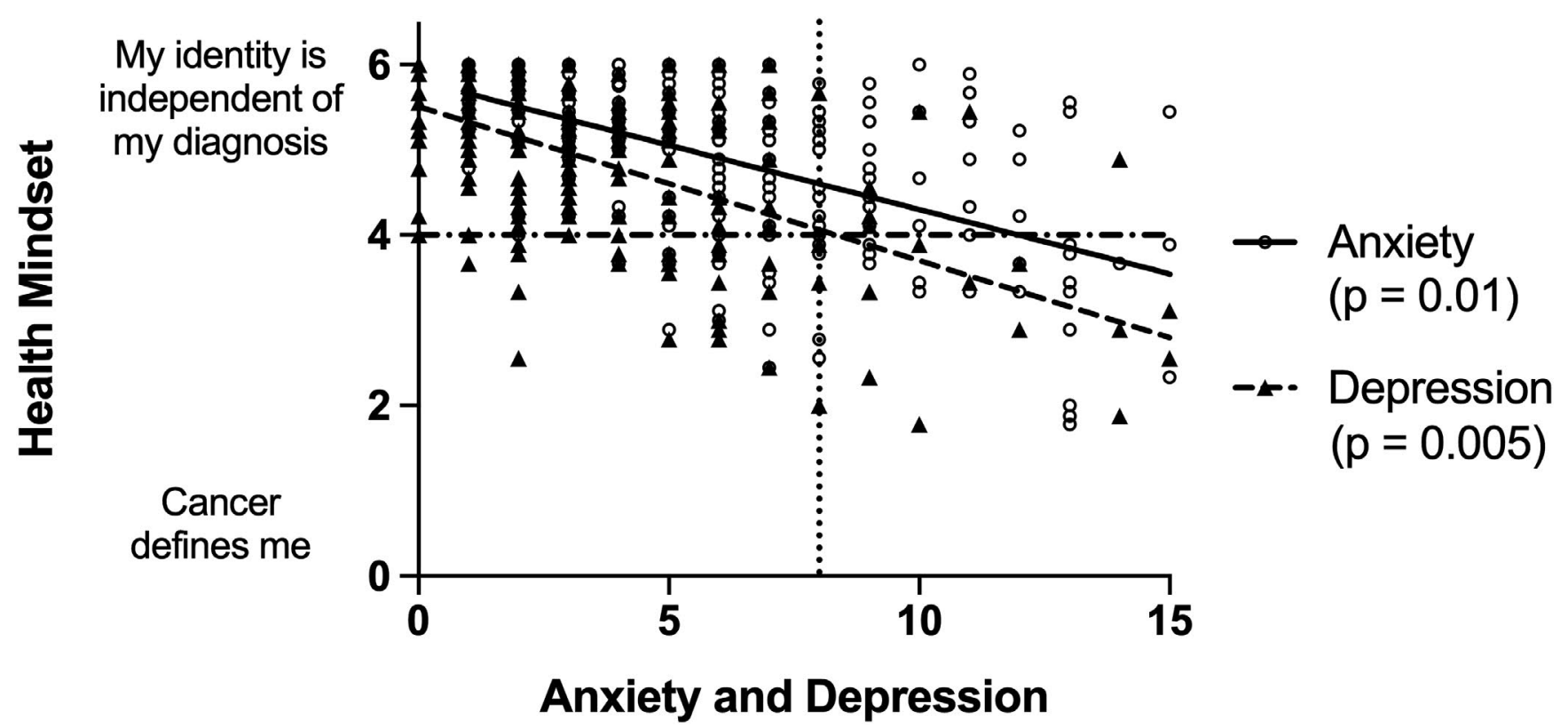

FIGURE 2 Health Mindset Instrument scores significantly correlated with anxiety $(P=.01)$ and depression $(P=.005)$ scores

\section{ORCID}

Paulina M. Gutkin (iD https://orcid.org/0000-0002-1359-0818

Kathleen C. Horst iD https://orcid.org/0000-0003-3883-8848

\section{REFERENCES}

1. Dweck CS. The choice to make a difference. Pers Psychol Sci. 2019;14(1):21-25.

2. Dweck CS. Mindsets: The New Psychology of Success. New York, NY: Random House LLC; 2006.

3. Zigmond AS, Snaith RP. The hospital anxiety and depression scale. Acta Psychiatr Scand. 1983;67(6):361-370.
4. Bastien $\mathrm{CH}$, Vallières $\mathrm{A}$, Morin $\mathrm{CM}$. Validation of the insomnia severity index as an outcome measure for insomnia research. Sleep Med. 2001;2(4):297-307.

5. Park CL, Zlateva I, Blank TO. Self-identity after cancer: "survivor", "victim", "patient", and "person with cancer". J Gen Intern Med. 2009;24(Suppl 2):S430-S435.

6. Thong MSY, Wolschon E-M, Koch-Gallenkamp L, et al. "Still a cancer patient"-associations of cancer identity with patient-reported outcomes and health care use among cancer survivors. J NCl Cancer Spectr. 2018;2(2):pky031. 